\title{
Erratum to: RAGE-binding S100A8/A9 promotes the migration and invasion of human breast cancer cells through actin polymerization and epithelial-mesenchymal transition
}

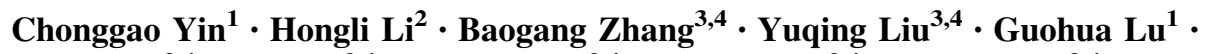
Shijun $\mathrm{Lu}^{3,4} \cdot$ Lei Sun ${ }^{3,4}$ Y Yueliang $\mathrm{Qi}^{3,4} \cdot$ Xiaolong $\mathrm{Li}^{3,4} \cdot$ Weiyi Chen ${ }^{3,4}$

Published online: 21 March 2016

(C) Springer Science+Business Media New York 2016

Erratum to: Breast Cancer Res Treat (2013)

142:297-309

DOI 10.1007/s10549-013-2737-1

In the original publication, the images in Fig. 3 were mistakenly selected from other experiments in which similar procedures were performed. The corrected Fig. 3 is given in this erratum.

The online version of the original article can be found under doi:10.1007/s10549-013-2737-1.

Baogang Zhang

zhangbg@wfmc.edu.cn; zbg0903@hotmail.com

1 College of Nursing, Weifang Medical University, Weifang 261053, China

2 Medicine Research Center, Weifang Medical University, Weifang 261053, China

3 Department of Pathology, Weifang Medical University, Weifang 261053, China

4 Department of Pathology, Key Clinical Specialty for Pathology of Shandong Province, Affiliated Hospital of Weifang Medical University, Weifang 261053, China 
Fig. 3 RAGE binding to S100A8/A9-induced EMT through the NF- $\kappa B$ signaling pathway. a The expression of E-cadherin and vimentin in $\mathrm{Scr} /$ MDA231, SiRAGE/MDA231, MCF-7/con, and MCF-7/RAGE cells with $10 \mu \mathrm{g} / \mathrm{mL}$ rS100A8/ A9 at different time points. $\beta$ actin was used as a loading control. Quantification of relative protein levels on three different western blots is shown below the blots. b Expression of epithelial markers, E-cadherin, as well as mesenchymal markers, $\mathrm{N}$-cadherin and vimentin, was examined by western blot in Scr/MDA231, SiRAGE/MDA231, MCF7/con, and MCF-7/RAGE cells with or without $10 \mu \mathrm{g} / \mathrm{mL}$ rS100A8/A9 for $48 \mathrm{~h}$. $\beta$-actin was used as a loading control. Quantification of relative protein levels on three different western blots is shown below the blots. c Fluorescence microscopic staining of E-cadherin, $\mathrm{N}$-cadherin, and vimentin (green) is indicated in the Scr/MDA231, SiRAGE/ MDA231, MCF-7/Con, and MCF-7/RAGE cells with rS100A8/A9 for 48 h. Nuclear DNA was stained with DAPI (blue). Scale bar $20 \mu \mathrm{m}$. Data were collected in this set of figures from a representative of at least three independent experiments

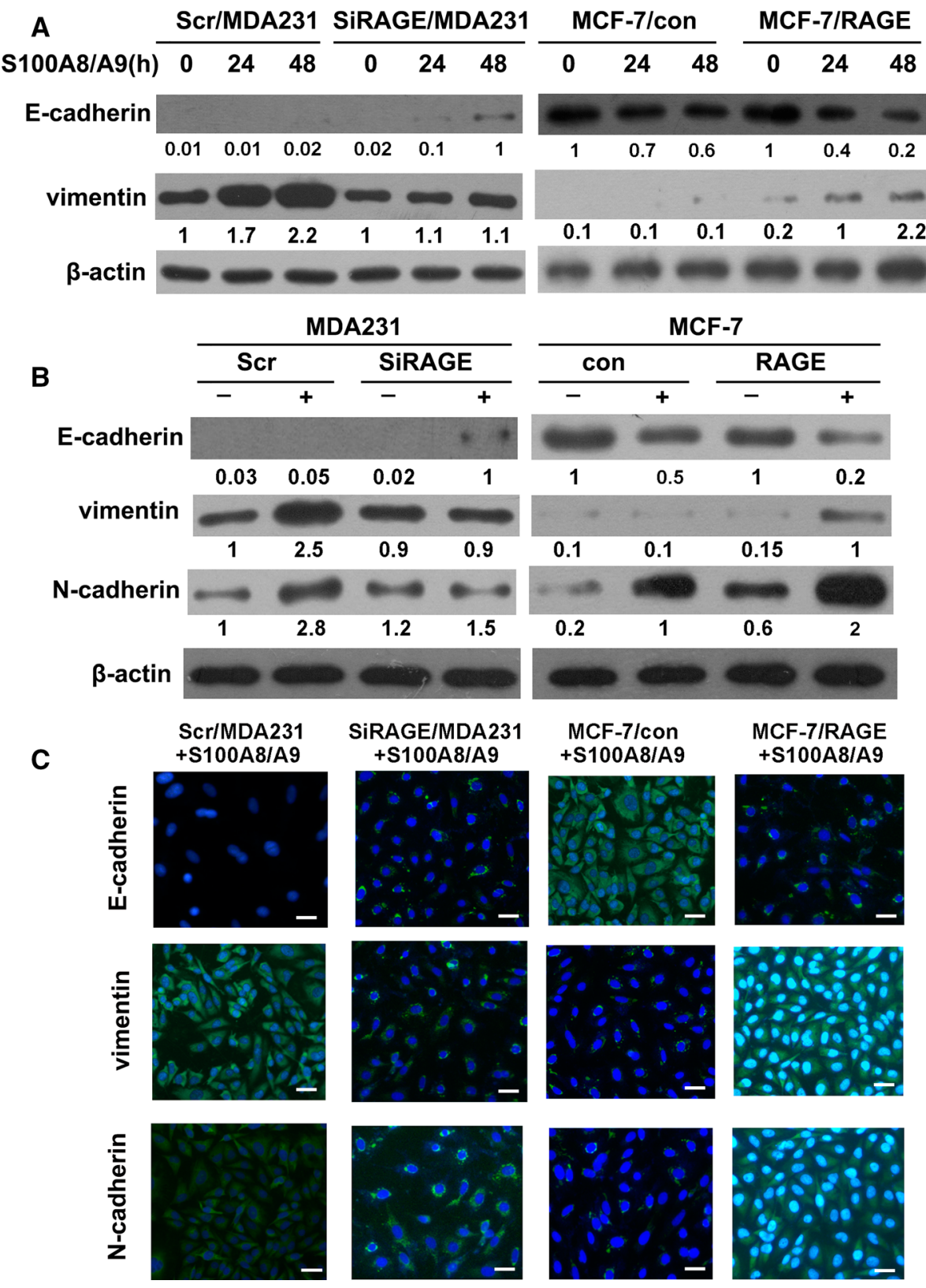

